\title{
Infant-parent psychotherapy at primary care level: Establishment of a service
}

\author{
Astrid Berg
}

Background. Access to infant and child psychiatric care in South Africa is limited. With focus on maternal and infant mental health, early identification and management of developmental, behavioural and psychosocial parent-child problems can be initiated.

Objectives. To establish a mental health service for children aged $0-3$ years, for delivery of infant-parent psychotherapy in a community setting.

Methods. The DC: 0-3R Classification for Mental Health and Development Disorders of Infancy and Early Childhood was used for diagnosis and standard multi-modal parent-infant psychotherapy methods for short-term interventions.

Results. A total of 179 infants and their maternal caregivers were seen. The primary referral symptom was faltering weight. The most frequent psychiatric diagnosis made was 'feeding disorder of caregiver-infant reciprocity'. Seventy two per cent of caregivers were exposed to more than 4 stress factors; $75 \%$ proved compliant with treatment with resultant improvement in the relationship between caregiver and child $(78 \%)$ and in the age-appropriated functioning of the infant $(76 \%)$.

Conclusion. This study confirms that a psychotherapeutic service for infants and mothers can be established at a primary healthcare level. Infant-parent psychotherapy was effective and readily accepted by the community. This service offers a training model for mental health providers enabling early recognition of mental health problems in children and psychotherapeutic intervention.

S Afr Med J 2012;102(6):582-584
Access to infant and child psychiatric care is limited in South Africa (SA), despite the fact that $1 / 5$ children and adolescents suffers from mental health disorders. ${ }^{1}$ In an attempt to rectify this deficiency, the Western Cape Department of Health embarked on an initiative in 2005 to establish comprehensive mental health services at primary healthcare and community levels. ${ }^{2}$

The Division of Child and Adolescent Mental Health at the University of Cape Town (UCT) established an infant mental health service at 2 sites in 1995: at a tertiary hospital and in a semiurban community on the outskirts of Cape Town. Establishment of the latter went through several phases: a site was selected where there was great need, negotiations with the local authorities were initiated and resources were made available. In 2006 the service was eventually located at a community clinic. It remains the only infant mental health service in the Western Cape.

The delivery of a mental health service to children aged 0 - 3 years in a community setting was a new concept; therefore, there were no precedents to follow. This is a report on the infant-parent psychotherapy service activities from 2006 to 2010. Ethical approval was obtained from the Faculty of Health Sciences Human Research Ethics Committee (HREC REF: 042/2011).

\section{Methods}

Children presenting for immunisation were weighed at every clinic visit. Infants and children aged $<3$ years who demonstrated faltering weight on the growth monitoring chart were referred to the infantparent psychotherapy service after medical screening to exclude acute physical pathology.

University of Cape Town and Red Cross War Memorial Children's Hospital, Cape Town

Astrid Berg, MB ChB, FCPsych (SA), MPhil (Child \& Adolescent Psychiatry)
Interviews and interventions were conducted by an Englishspeaking child psychiatrist and a Xhosa-speaking cultural counsellor and were performed in the home language of the participant. Basic demographics assessed included age, sex, and family constellation, presenting problems, clinical observation of the mother-infant interaction, and the infant's affective, cognitive and social interactions. Prospective and observational semi-structured interviews and therapeutic sessions were conducted with the infants and their caregivers utilising Maldonado-Duran's multimodal parent-infant psychotherapy method and Lieberman's childparent psychotherapeutic model. ${ }^{3,4}$ For diagnosis the DC: 0-3R Diagnostic Classification of Mental Health and Developmental Disorders of Infancy and Early Childhood was used. This is a multi-axial diagnostic classification system used for the psychiatric assessment of children aged $<3$ years. It embraces the Clinical Disorder of the infant (Axis I), the Relationship Classification (Axis II) emphasising the fundamental importance of the parent-infant relationship, Medical and Developmental Disorders and Conditions (Axis III), Psychosocial Stressors (Axis IV) and Emotional and Social Functioning of the infant (Axis V). ${ }^{5}$

Criteria used for assessing Axis II were the observed behaviour of the caregiver and infant, the caregiver's subjective experience of the infant, and the experience of the infant during the interview through the observation of play with an object. Axis V was assessed on a 6-point rating scale focused on attention and regulation of affect, mutual engagement and intentional two-way communication. ${ }^{5}$

The mood state of caregivers and infants was observed and documented. A caregiver's depressed mood was diagnosed through symptoms of sadness, tiredness, lack of energy, low self-esteem and lack of pleasure, during interaction with the child. ${ }^{6}$ Depression in the infants was defined as weight loss, general withdrawal, lack of engagement with the caregivers and the environment, little or no spontaneous play, and 'sad affect'.

Information from initial interviews formed the basis for shortterm multimodal infant-parent psychotherapeutic intervention targeted at identified stressors. Additional psychotherapeutic sessions were scheduled as required, depending on patient load and individual need. With the aim of strengthening the relationship between 
caregiver and infant, these sought to assist the caregiver in mobilising emotional and physical resources to overcome barriers interfering with emotional investment in the child.

\section{Results}

A total of 179 infants were seen from 2006 to 2010, including 80 males and 99 females. All were from Xhosa-speaking families in Khayelitsha. Age at referral ranged from 1 to 41 months (mean 15.6 months).

Family constellation data showed the mother as the sole caregiver in 47 infants and toddlers, with the maternal grandmother fulfilling this role in 13, and both in 32 . Of the 179 caregivers, 95 mothers expressed negative feelings (absence, no financial support, domestic violence) towards the father. Seven children were in foster care.

The primary referral symptom was faltering weight. Teenage pregnancies, maternal psychiatric illness and family bereavement were regarded as additional risk factors prompting referral. Axis I of the DC: 0 -3R (Table 1) was utilised for diagnosis of clinical disorders in the infants. The main psychiatric diagnosis was feeding behaviour disorders and 2 categories were added. Despite lengthy interviews, no discernible clinical psychiatric disorder could be identified in 31 infants.

Concurrent medical conditions (Axis III) were seen in 41 and included HIV infections in 17, gastro-enteritis in 8, developmental delay in 12 and cardiac, eye, and congenital abnormalities in 4 .

Axis IV provided a framework for the identification and evaluation of the psychosocial and environmental stressors; 129 caregiver-infant pairs were exposed to 4 or more multiple simultaneous psychosocial stressors. These included challenges to the child's primary support, social environment, child care, housing and economic circumstances.

A depressed maternal mood was observed in 63 and infant withdrawal in 51 . This was particularly prevalent in the pairs classified under 'feeding disorder of caregiver-infant reciprocity', with 33 mothers and 24 infants suffering from a depressed mood.

A total of 134 mothers were compliant with treatment $(2-25$ sessions, mean 4.7), receiving psychotherapeutic intervention until there was improvement; 37 were eventually referred to medical or social services.

Of the last 55 patients seen, 43 infants (78\%) maintained or

Table 1. Axis I: Infant clinical disorders according to DC: 0-3R

\begin{tabular}{ll}
\hline No psychiatric diagnosis & 31 \\
100. Post-traumatic stress disorder & 6 \\
200. Disorders of affect & 1 \\
210. Prolonged bereavement/grief reaction & 1 \\
300. Adjustment disorder & 1 \\
601. Feeding disorder of state regulation & 5 \\
602. Feeding disorder of caregiver-infant reciprocity & 66 \\
603. Infantile anorexia & 1 \\
605. Feeding disorder associated with concurrent medical & \\
$\quad$ condition & 41 \\
$\quad$ Food insecurity & 19 \\
$\quad$ Dietary misinformation & 2 \\
700. Disorders of relating and communicating & 3 \\
800. Other disorders & 1 \\
Unknown & 1 \\
Total & 179
\end{tabular}

Total improved their adapted and disordered relationships, respectively (Axis II). Fifteen pairs failed to make substantial improvement: 7 infants were developmentally delayed, including 1 with autism. In 13, 4 or more stressors were operative and in 10/15 households, a paternal figure was absent.

Of the 55 infants, $42(76 \%)$ remained functional at an ageappropriate level on the Emotional and Social Functioning scale (Axis V); 13 (56\%) improved from an 'at-risk' rating to an ageappropriate rating, i.e. their attention and regulation of affect, mutual engagement and intentional two-way communication improved to within the normal range.

Those mothers and infants who had the greatest number of ongoing sessions either achieved or maintained their optimal functioning.

\section{Discussion}

Primary healthcare has shifted from a curative, hospital-based system to one that emphasises prevention and health promotion. ${ }^{7}$ Emphasis on the mental health of mothers and infants is in line with the Alma Ata Declaration of 1978: early intervention, increased awareness of mental health from the start of life, and a holistic approach to care.

An individual psychotherapeutic approach is not part of standard practice in community clinics which are centred on group sessions for mothers with a common problem (e.g. HIV). A new therapeutic approach had to be developed as there were no examples of practical assistance in SA.

The availability of a confidential space with a consistent and reliable clinical team - allowing for individual and personal connection was important, particularly within the community context where anonymity and privacy are not easily attained. ${ }^{7}$ Embedded in the supportive psychotherapeutic approach was 'unstructured reflective developmental guidance' as described by Lieberman. ${ }^{4}$ The clinical team responded to the needs of the moment, rather than following a prescribed treatment module; it was considered reflective because the parent was encouraged to think about the infant and find a different way of viewing their situation. Bestowing personhood onto the infant through talking to the infant made them an active partner in the interaction. ${ }^{8}$ This was an important modelling action for mothers whose sense of competence in their maternal abilities was impaired. Crisis intervention and case management were indicated, particularly in cases of domestic violence. Concrete advice and referrals to appropriate agencies were given.

Failure to thrive, or faltering growth, was an obvious sign, compounded by the caregiver's feelings of hopelessness and helplessness and the burden of real-life problems and multiple stressors. Maternal depressive mood was associated with poor infant growth, resulting in a greater risk to the child's physical and mental wellbeing. ${ }^{9}$ Given the referral bias to the service, it was not unexpected that infant withdrawal was present in $28 \%$ and maternal depressed mood in 35\% (mirroring the findings of Cooper et al. ${ }^{10}$ of $34.7 \%$ in mothers in an equivalent environment). Depressive mood was particularly prevalent among mothers of infants with the diagnosis of 'feeding disorder of caregiver-infant reciprocity'.

In infant psychopathology, medical and social conditions that impact on the child may require referral to appropriate services as was the case in $21 \%$ of patients.

There was a high rate of return visits $(75 \%)$ on the part of mothers who expressed appreciation of the emotional support that the pair received. In contrast, $25 \%$ of pairs defaulted treatment; these caregivers were particularly compromised in terms of domestic violence, alcohol abuse, teenage motherhood and major psychiatric disorders, contributing to their classification under Disordered Relationship in Axis II. 
A disquieting finding was that more than half of the children lived in father-absent/female-headed households. Even when fathers were physically present, many mothers admitted to a negative relationship with their partners. These findings together have potentially serious implications for the socio-emotional development of the children.

However, single-parent female-headed households are not necessarily ineffective as shown by the favourable responses to psychotherapeutic interventions. Seventy-three per cent maintained their adapted relationship or improved their disordered relationship with their infants, ultimately to the benefit of the child (Axis II). Significantly, in $56 \%$ of pairs the infants improved from at-risk to age-appropriate level functioning (Axis V), showing the resilience inherent in the infants who responded positively to the improved relationship.

While caregivers may have been materially poor, they were not necessarily psychologically impoverished; it would have been pejorative to assume that they needed to be told 'what to do' for the infants. The inhibition of (grand-)mothering skills had to do with pre-occupations and negative effects of their many life stressors. Once these were dealt with, often through minimal psychotherapeutic input, they were freed to do what was best for infants in their care, through, it might be postulated, enhancement of their sense of selfefficacy: 'the belief in one's ability to successfully perform a particular behavior. ${ }^{11}$ This has been shown to moderate the relation between stressful conditions and coping/performance accomplishments, as testified by a spontaneous comment from a grandmother: 'Things are much better, no longer so serious. I am able to think and think of solutions; coming here has helped ... I feel like a person amongst other people. Even if I walk, I walk upright - from coming here.'

\section{Conclusions}

Infant and maternal mental health provides a natural entry point for the development of decentralised, quality psychiatric services at primary healthcare level. The findings of this study emphasise the dependence of the physical well-being of infants on the mental wellbeing of their mothers or other caregivers. Faltering growth can be used by primary healthcare nurses as an indicator for infant mental health vulnerability. Self-efficacy was made possible by offering mothers and infants the opportunity to reflect on their lives, and through concrete advice and guidance. Multiple socio-economic stressors and language differences need not be regarded as barriers to successful child-parent psychotherapy.
The study suggests the possibility of training primary healthcare workers to recognise signs of emotional problems and to understand and apply the basic principles of psychotherapy. ${ }^{12}$ Emphasis should be on continuity, consistency, confidentiality and open-mindedness, with a proper understanding of community norms, values and practices.

This new collaborative model of a child psychiatrist and primary healthcare worker permits early recognition and management of infant mental health disorders. ${ }^{13,14}$ This meets the requirements for primary preventive healthcare and is a response to the 'Call for action' articulated by the Lancet Global Mental Health Group. ${ }^{15}$

Acknowledgements. The author wishes to thank Nosisana Nama (cultural counsellor).

\section{References}

1. Kleintijes S, Lund C, Flisher AJ, MHAPP Research Programme Consortium. A situational analysis of child and adolescent mental health services in Ghana, Uganda, South Africa and Zambia. Afr J Psychiatry 2010;13(2):132-139.

2. Western Cape Department of Health (DoH). Comprehensive Service Plan for the Implementation of Health Care 2010. Cape Town:Western Cape DoH, 2007.

3. Maldonado-Duran J, Lartigue T. Multimodal parent-infant psychotherapy. In: Maldonado-Duran J, ed. Infant and Toddler Mental Health.Washington, DC: American Psychiatric Publishing Inc, 2002:129-160.

4. Lieberman AF, Van Horn P. Child-parent psychotherapy: A developmental approach to mental health treatment in infancy and early childhood. In: Zeanah $\mathrm{CH}$, ed. Handbook of Infant Mental Health. 3rd ed. New York, London: The Guildford Press; 2009:439-449.

ed. New York, London: The Guildford Press; 2009:439-449.
ZERO TO THREE. Diagnostic Classification of Mental Health and Developmental Disorders of Infancy 5. ZERO TO THREE. Diagnostic Classification of Mental Health and Developmental Disorders of Inf
and Early Childhood: Revised edition (DC:0-3R). Washington, DC: ZERO TO THREE Press, 2005.

6. Patel V, Rahman A, Jacob KS, Hughes M. Effect of maternal mental health on infant growth in low income countries: new evidence from South Asia. BMJ 2004;328:820-823.

7. Mkhize N, Kometsi MJ. Community access to mental health services: lessons and recommendations. South African Health Research Journal 2008;103-113.

8. Berg A. Talking with Infants: A bridge to cross-cultural intervention. Southern African Journal of Child and Adolescent Mental Health 2002;14(1):5-14. [http://dx.doi.org/10.1080/16826108.2002.9632419]

9. Rahman A, Harrington R, Bunn J. Can maternal depression increase infant risk of illness and growth impairment in developing countries? Child: Care, Health and Development 2002;28:51-56. [http:// dx.doi.org/10.1046/.1365-2214.2002.00239.x]

10. Cooper PJ, Tomlinson M, Swartz L, Woolgar M, Murray L, Molteno C. Post-partum depression and the mother-infant relationship in a South African peri-urban settlement. Br J Psychiatry 1999;175:554558. [http://dxdoiong $10.1192 / b i$. 175.6554$]$

11. Coleman PK, Karraker KH. Self-efficacy and parenting quality: Findings and future applications. Dev Rev 1997;18:47-85. [http://dx.doi.org/10.1006/drev.1997.0448]

12. Knapp PK, Ammen S, Arstein-Kerslake C, Poulsen MK, Mastergeorge A. Feasibility of expanding services for very young children in the public mental health setting. J Am Acad Child Psy 2007;46(2):152-161. [http://dx.doi.org/10.1097/01.chi.0000246058.68544.35]

13. Connor DF, McLaughlin TJ, Jeffers-Terry M, et al. Targeted child psychiatric services: A new model of pediatric primary clinician-child psychiatric collaborative care. Clin Pediatr 2011;45(5):423-434. [http://dx.doi.org/10.1177/0009922806289617]

14. Walker S. A description of the establishment of a new child and adolescent mental health service in the United Kingdom. J Child Adol 2010;22(1):35-39. [http://dx.doi.org/10.2989/17280583.2010.493664]

15. Lancet Global Mental Health Group. Scale up services for mental disorders: a call for action. Lancet 2007;370:1241-1252. [http://dx.doi.org/10.1016/S0140-6736(07)61242-2]

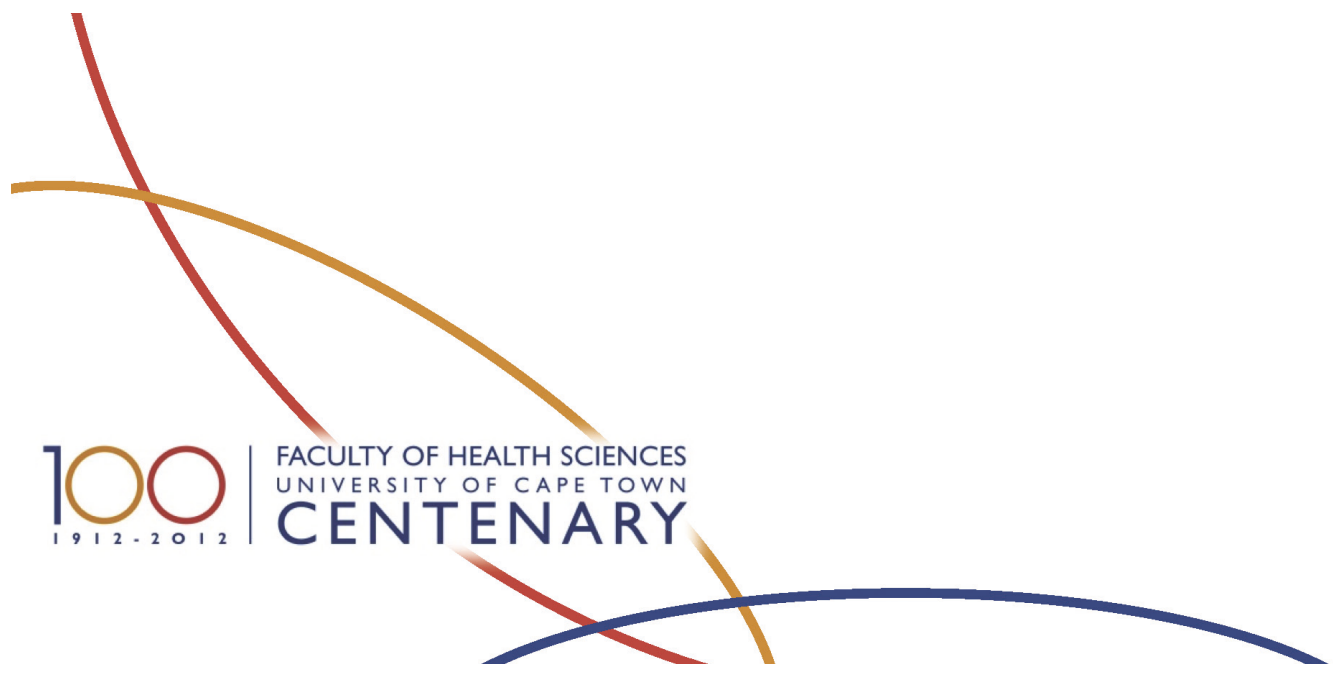

